\title{
Variaciones Vasculares en Cavidad Abdominal y de Vías Urinarias. Presentación de Caso
}

\author{
Vascular Variations in Urinary Tract and Abdominal Cavity. Case Report
}

Ballesteros, L. E.*; Estupiñan, H. Y.* \& Ballesteros, D. R.*

\begin{abstract}
BALLESTEROS, L. E.; ESTUPIÑAN, H. Y. \& BALLESTEROS, D. R. Variaciones vasculares en cavidad abdominal y de vías urinarias. Presentación de caso. Int. J. Morphol., 34(1):404-409, 2016.

RESUMEN: Se presenta un raro caso de múltiples variaciones en la cavidad abdominal de un espécimen cadavérico de 50 años de género masculino, del laboratorio de anatomía de la Universidad Industrial de Santander (Bucaramanga-Colombia). Se observó variaciones arteriales (arteria renal adicional derecha y origen de la rama hepática derecha desde la arteria mesentérica superior), venosa (vena renal derecha adicional) y de vía urinaria (doble uréter en el lado derecho). Estas diversas variantes anatómicas además de suscitar interés académico, deben ser consideradas y descritas correctamente por los clínicos durante la realización de procedimientos quirúrgicos, radiológicos y de imágenes diagnósticas en la cavidad abdominal.
\end{abstract}

PALABRAS CLAVE: Arteria renal derecha; Vena renal derecha; Rama derecha de arteria hepática; Uréter bífido.

\section{INTRODUCCIÓN}

Las variaciones vasculares en la cavidad abdominal junto con los de las vías urinarias son relativamente frecuentes, pero en los diferentes estudios se referencian por separado, por lo que se ignora la incidencia en que dos o más expresiones variantes se presentan en un mismo individuo (Holden et al., 2005; Zhu et al., 2015; Satyapal, 2003; Vilhova et al., 2001; Koops et al., 2004). Dentro de las variaciones arteriales se destacan la presencia de arterias renales adicionales (ARA) y las variaciones en el origen de las ramas hepáticas. La ARA ha sido reportada en un rango de 13,5-40 \% (Khamanarong et al., 2004; Saldarriaga et al., 2008). Igualmente, del patrón usual de las ramas hepáticas derecha e izquierda originadas de la arteria hepática común, se presenta una expresión variante en donde, la rama hepática derecha (RHD) se origina de la arteria mesentérica superior, variación reportada entre el 6,2-18 \% (Hiatt et al., 1994; Michels, 1966).

En diferentes grupos poblacionales también se ha descrito la presencia de venas renales adicionales (VRA) con una incidencia de 8-38,5\%, las cuales se presentan en el $98 \%$ de los casos en el lado derecho y sin diferencias significativas de sexo (Ballesteros et al., 2014; Beckmann \& Abrams, 1980; Kawamoto et al., 2005). Dentro de las variaciones de las vías urinarias, la duplicidad de los uréteres es la expresión más frecuente; en estos casos, los cálices mayores no confluyen y dan origen a dos uréteres, medial y lateral que cursan longitudinalmente sobre la pared abdominal posterior, uniéndose al ingresar a la cavidad pélvica (Anderson \& Cadeddu, 2012). La información existente en la literatura sobre las diversas expresiones vasculares y de las vías urinarias se enriquece de los estudios sobre una variación en particular; mientras que las variaciones múltiples observadas en un individuo deben reportarse como casos. El buen conocimiento sobre estas variaciones impacta a los procesos de enseñanza-aprendizaje de la anatomía y permite mejorar los abordajes abdominales, especialmente los de urgencias, en los que estas estructuras están comprometidas, reduciéndose de esta manera las iatrogenias al realizar estos procedimientos quirúrgicos.

\section{REPORTE DE CASO}

Cadáver de sexo masculino de 50 años, perteneciente al laboratorio de morfología de la Universidad Industrial de Santander, fijado con solución fijadora conservadora chilena. Se realizó el abordaje de cavidad abdominal, mediante una incisión en línea mediana, desde el proceso xifoideo hasta el pubis, con separación de los colgajos fascio-musculares derecho e izquierdo; luego, se disecaron por planos las 


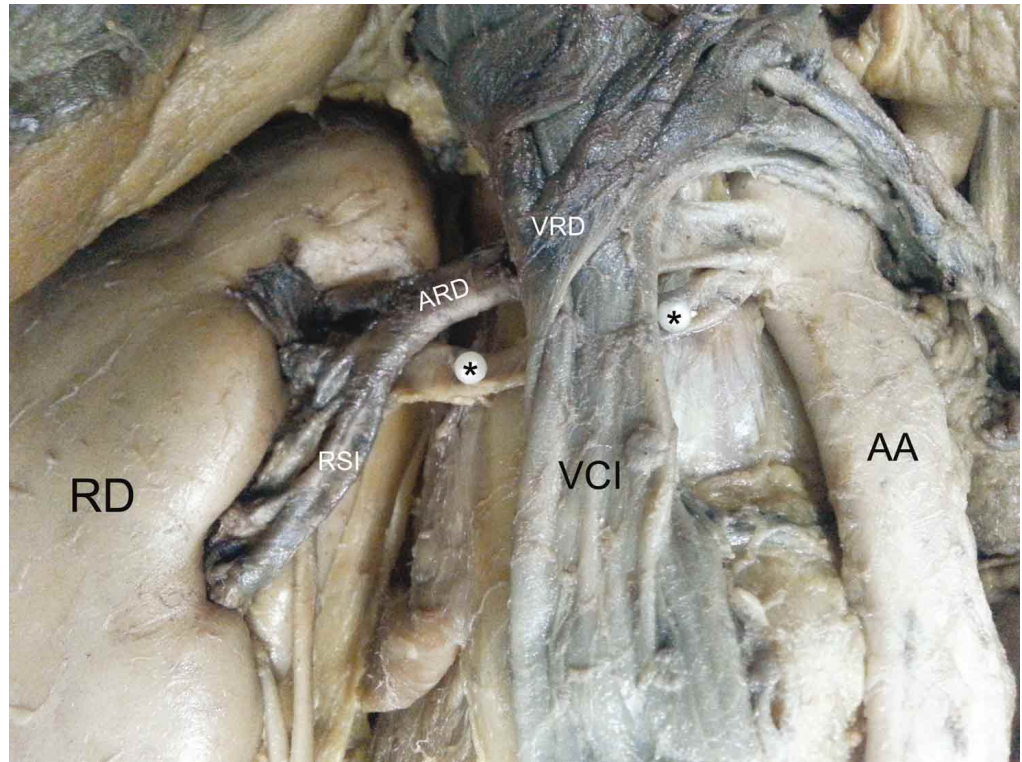

Fig. 1. Arteria renal adicional lado derecho. RD. Riñón derecho; VCI. Vena cava inferior; AO. Aorta abdominal; ARD. Arteria renal derecha; RSI. Rama segmentaria inferior; VRD: Vena renal derecha que se ha reclinado para permitir la observación del componente arterial. (*) Arteria renal adicional.

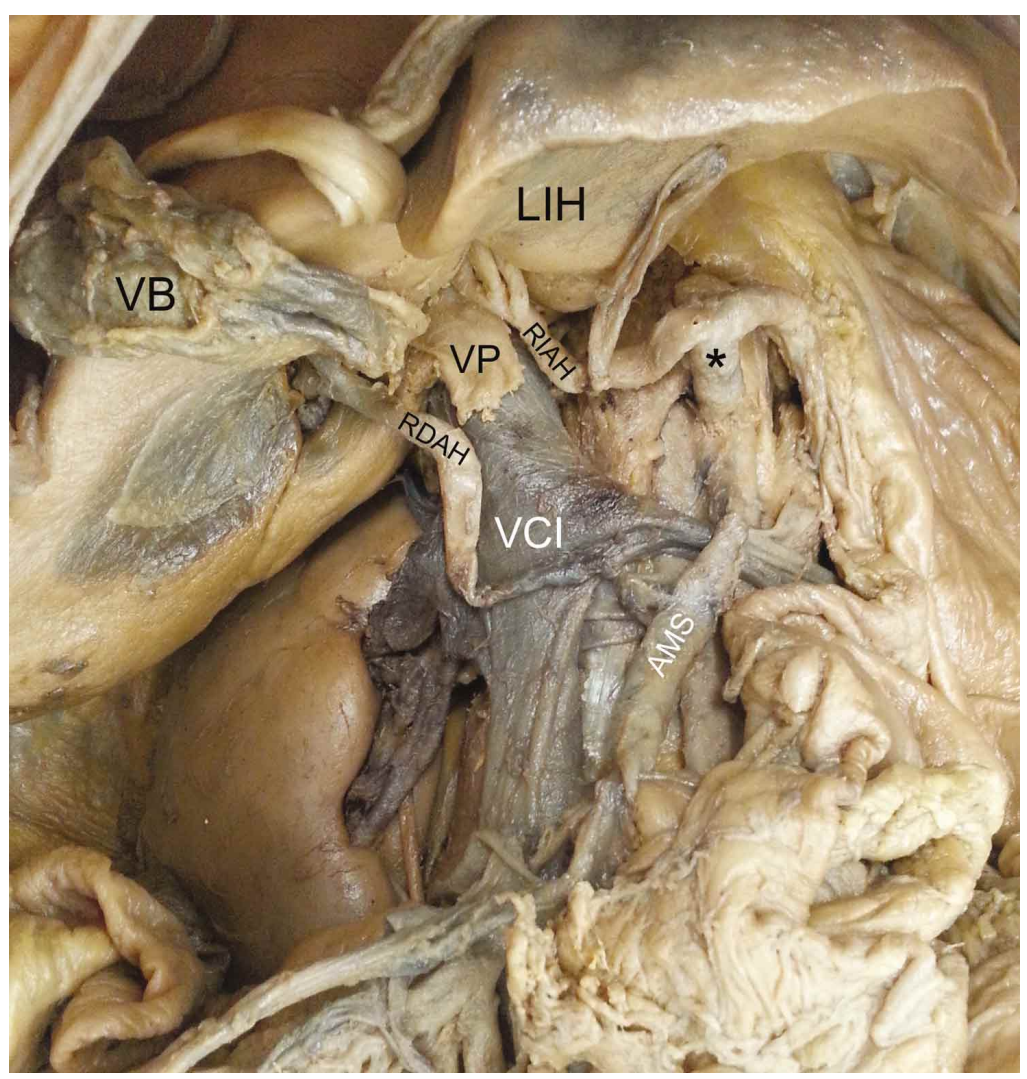

Fig. 2. Rama hepática derecha originada de la arteria mesentérica superior. VB. Vesícula biliar; LIH. Lóbulo izquierdo del hígado; VCI. Vena cava inferior; VP. Vena porta seccionada; RIAH. Rama izquierda de la arteria hepática; RDAH. Rama derecha de la arteria hepática; AMS. Arteria mesentérica superior; $(*)$ tronco celiaco. estructuras, musculares y vasculares de la pared abdominal y mediante incisión nivel del reborde costal se dejó expuesta la cavidad abdominal, y se procedió a la disección de las estructuras peritonizadas y retroperitoneales.

Se hallaron las siguientes variaciones anatómicas:

Arteriales. Se observó en el lado derecho una ARA. La arteria renal principal se originó de la superficie lateral de la parte abdominal de la aorta a nivel de L1, presentó un calibre proximal de 5,9 $\mathrm{mm}$. Este vaso adoptó una trayectoria horizontal, pasando por detrás de la vena cava inferior y cerca al hilio renal dio origen a las ramas segmentarias apical, anterosuperior, antero-inferior e inferior. La ARA se originó de un ostium adyacente e inferior a la arteria renal principal. Con un calibre proximal de 3,7 mm y una longitud de 52,9 mm, cursó con una trayectoria paralela a la arteria principal e ingresó al hilio renal por detrás del cáliz mayor superior (Fig. 1).

La arteria hepática común con un calibre de 7,5 mm y una longitud de 19,6 mm, dio origen a la arteria gastroduodenal y a la arteria hepática izquierda que en el hilio hepático dio origen a dos pequeñas ramas intralobares. A $6,7 \mathrm{~mm}$ del tronco celiaco se originó la arteria mesentérica superior con un calibre proximal de 9,3 mm. Una vez la arteria mesentérica cursó anterior a la tercera porción del duodeno dio origen a la RHD que adoptó una trayectoria ascendente incorporándose al pedículo hepático, en donde tomó trayectoria paralela a la vía biliar y originó en su segmento distal a la rama cística; antes de penetrar al hilio, se bifurcó en ramas intra-lobares anterior y posterior que presentaron calibres de 3,0 y 2,3 mm respectivamente. La longitud de la RHD fue de 80,2 mm y su calibre 5,2 mm (Fig. 2).

Venosas. En el lado derecho se observó una VRA originada de una vena tributaria superior que presentó una longitud de 27,2 mm y un calibre de 8,2 mm; cursó con una trayectoria ligeramente oblicua y paralela a la vena renal derecha principal, drenando en la vena cava inferior al nivel de su superficie lateral. La VRA se ubicó en un plano antero-superior con relación a las dos arterias renales. 


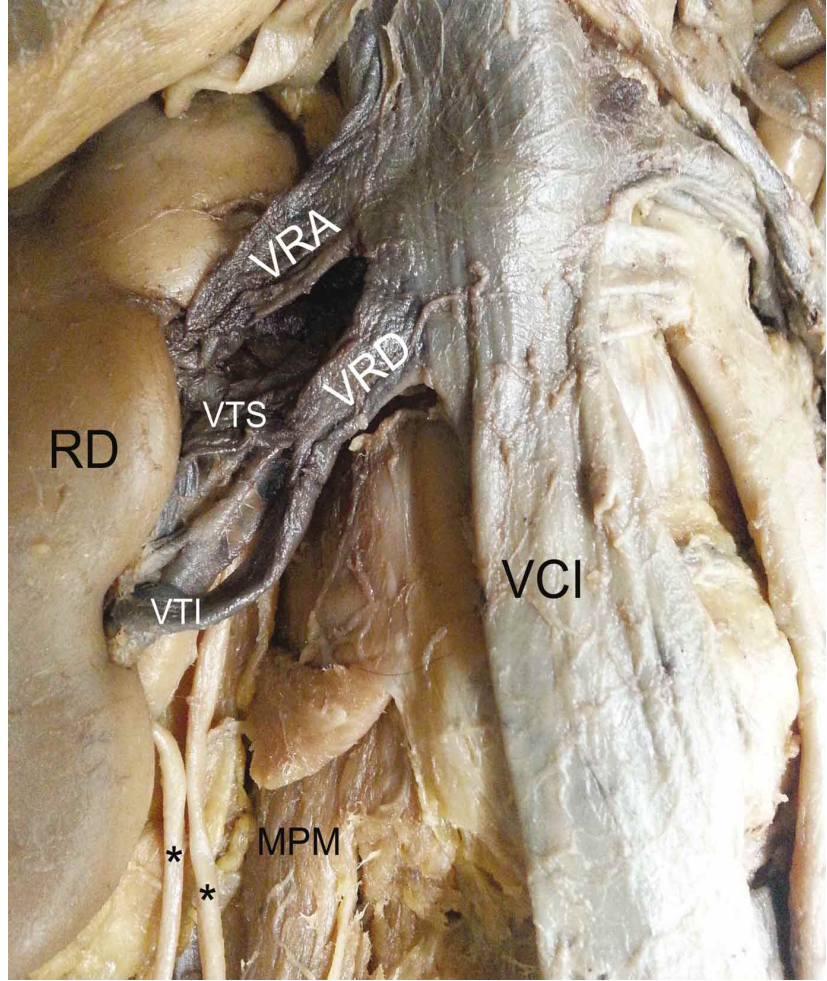

Fig. 3. Vena renal adicional lado derecho. RD. Riñón derecho; VCI. Vena cava inferior; MPM. Musculo psoas mayor; VRA. Vena renal adicional; VRD. Vena renal derecha; VTS. Vena tributaria superior, VTI: Vena tributaria inferior. (*) Uréter bífido.

La distancia entre las dos venas renales derechas al nivel de su segmento medio fue $9,1 \mathrm{~mm}$. La vena renal derecha principal se originó de las tributarias superior (longitud: $20,1 \mathrm{~mm}$; calibre: $4,9 \mathrm{~mm}$ ) e inferior (longitud: $28,6 \mathrm{~mm}$; calibre: $5,9 \mathrm{~mm}$ ), las cuales se unieron en un plano extrahiliar. La vena renal principal presentó una longitud de $22,4 \mathrm{~mm}$ y un calibre de $8,4 \mathrm{~mm}$ (Fig. 3).

Vías urinarias. En el lado derecho se observó uréter bífido con convergencia a $29,7 \mathrm{~mm}$ de la pared vesical posterior. El uréter medial con calibre de $3,7 \mathrm{~mm}$ se encontró como continuación del cáliz mayor superior a $22,7 \mathrm{~mm}$ de distancia del uréter lateral en relación con el hilio renal, mientras que el uréter lateral con calibre de $3,5 \mathrm{~mm}$ surgió de la unión de los cálices mayores medio e inferior. El uréter medial, al nivel del hilio renal se ubicó por delante de la ARA. Los dos uréteres cursaron en paralelo sobre la superficie del músculo psoas mayor manteniendo esta disposición a su paso sobre los vasos iliacos. El riñón derecho (longitud: 121,9 mm; ancho: 57,5 mm; grueso: 36,0 $\mathrm{mm}$ ) con la bifurcación del uréter presentó diferencias morfométricas con relación al riñón izquierdo (longitud: 99,2 mm; ancho: 57,1 mm; espesor: 38,2 mm) (Fig. 4).

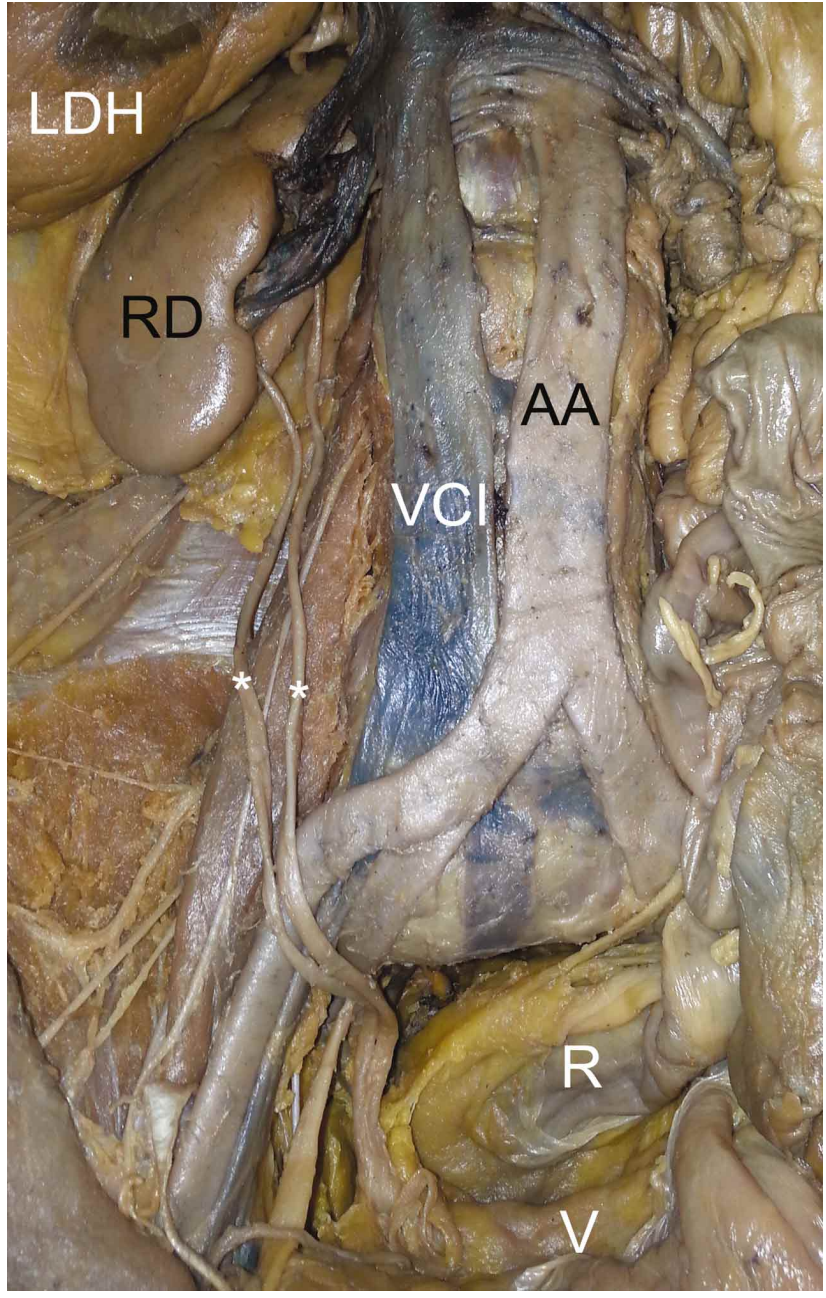

Fig. 4. Uréter bífido lado derecho. RD. Riñón derecho; VCI. Vena cava inferior; AO. Aorta abdominal; LDH. Lóbulo derecho del hígado; V. Vejiga; R. Recto; (*) Uréter bífido.

\section{DISCUSIÓN}

La presencia de VRA ha sido reportada en un amplio espectro de variabilidad en un rango de 8-38,5\%. Las incidencias más altas han sido reportadas en un rango de 33-38,5 \% (Beckmann \& Abrams; Holden et al.), mientras que otros autores han informado la presencia de esta variación en el 18-26\%, en estudios llevados a cabo en diversos grupos de poblaciones (Ballesteros et al.; Satyapal et al. 2001; Zhu et al.). Existe pleno acuerdo entre los diferentes estudios que señalan baja frecuencia de venas renales adicionales del lado izquierdo 1,5\% (Costa et al., 2011) y 2,6 \% (Satyapal).

La amplia variabilidad en la incidencia de VRA reportada en la literatura se debe probablemente a factores como el tamaño de la muestra, o a las características biológicas que determinan la expresión variable de estas estructuras en los di- 
versos grupos de población evaluados. Igualmente, las metodologías utilizadas para determinar sus características como la disección directa o la utilización de imágenes pueden afectar los resultados de estos hallazgos (Beckmann \& Abrams; Ballesteros et al.; Zhu et al.). Es de destacar que, debido a la dificultad de obtener un número adecuado de muestras de sujetos femeninos, los estudios anatómicos directos tienen evidentes limitaciones para correlacionar sus resultados por sexo. Las variaciones en el número de venas renales son clínicamente silentes y pueden pasar inadvertidas hasta que se descubren durante un procedimiento quirúrgico o en la necropsia. Su presencia adquiere gran importancia en el trasplante renal, debido a que influyen en la viabilidad técnica de la operación, por lo que se requiere su evaluación mediante venografía preoperatoria (Satyapal; Zhu et al.; Kawamoto et al.).

La longitud de la vena renal derecha (VRD) reportada en estudios angiográficos, de disección directa y de inyección de los lechos vasculares se encuentra en un rango de 23-28 mm (Satyapal; Janscheck et al.,; Ballesteros et al.) concordante con nuestro hallazgo. Las diferencias señaladas en los reportes de la longitud de las venas renales se deben principalmente a que estas estructuras se originan al nivel hiliar o extrahiliar. El origen extrahiliar observado en el presenta caso es reportado en estudios previos entre 25-28 \% (Holden et al.; Janscheck et $a l$.). El origen extra-hiliar de las venas renales deja a sus vasos tributarios más expuestos, por lo que estas estructuras quedan más vulnerables al trauma y a las lesiones durante los procedimientos quirúrgicos. El calibre de la VRD de este reporte $(9,1$ $\mathrm{mm}$ ) es menor a lo informado $(12-13 \mathrm{~mm})$ por Satyapal, Janscheck et al. y Beckmann \& Abrams en estudios de venas renales únicas, pero es concordante con estos autores la presencia de calibres para las venas adicionales dentro de un rango de 5-9 mm. En cuanto a la configuración de las tributarias de la VRD, la presencia de dos tributarias, superior e inferior, encontrado en nuestra serie, es la más frecuentemente reportada (55-65\%) por Ballesteros et al. y Costa $e t a l$.

La presencia de ARA ha sido reportada en un rango de 13,5-40 \%. Las incidencias más bajas (13,5-18 \%) se reportan en indios, tailandeses y negros (Khamanarong et al.; Satyapal), mientras que las incidencias medias y altas se han reportado en mestizos colombianos y en caucásicos (Saldarriaga et al.; Vilhova et al.).Se presenta discrepancia entre los diversos estudios cuando se evalúa el lado de presentación de las ARA : algunos autores han informado de una frecuencia más alta en el lado izquierdo (Satyapal; Vilhova et al.) mientras que en otros trabajos esta variación es más frecuente en el lado derecho (Khamanarong et al.; Cicekcibasi et al., 2005) pero sin que estas diferencias sean estadísticamente significativas. Se ha reportado presencia de ARA bilaterales en el 4,3-10,2\% (Cicekcibasi et al.; Satyapal et al.). Pocos estudios anatómicos han descrito de manera detallada las trayectorias paralelas ho- rizontales, o cruzadas divergentes de las arterias adicionales con relación a la arteria renal principal (Saldarriaga et al.; Cicekcibasi et al.). Por su sitio de ingreso al parénquima renal pueden ser hiliares, como la observada en este caso y reportadas en el 60-70 \% o polares superiores o inferiores (Saldarriaga et al.; Cicekcibasi et al.).

La gran variabilidad en la trayectoria de la arteria adicional, su distancia al origen de la arteria renal principal y el nivel de ramificación en vasos segmentarios, son aspectos que deben tenerse en cuenta durante procedimientos quirúrgicos que involucran los riñones y el retroperitoneo. La ramificación precoz de la arteria renal principal puede ser erróneamente interpretada como arteria adicional en los estudios de diagnóstico por imagen o determinar complicaciones quirúrgicas en el trasplante renal, ya que los primeros $15 \mathrm{~mm}$ de la arteria renal pueden ser utilizados para anastomosis con la arteria ilíaca del destinatario. También hay que destacar que la ramificación temprana de la arteria renal principal y la presencia de ARA se constituyen en criterios de exclusión para la realización de cirugía laparoscópica renal. Tener en cuenta estas consideraciones anatómicas se reflejará por tanto, en una franca reducción de complicaciones al realizar estos procedimientos (Holden $e t$ al.; Janschek et al.; Vilhova et al.).

Las longitudes de las arterias renales derecha principal y adicional ( 38,5 y $42,8 \mathrm{~mm}$ ) observado en el presente caso son concordantes con lo reportado por Satyapal. Una mayor longitud de la ARD (44 mm) fue informada por Janschek et al.. El nivel de la ramificación de las arterias renales, cerca o lejos del hilio renal es el factor que determina la longitud de estos vasos, que pueden ser rasgos morfológicos de los diferentes grupos poblacionales. Las arterias renales cortas, con 10-25 mm de longitud han sido reportadas con un incidencia de 8,3-19 \% (Saldarriaga et al.; Holden et al.; Kawamoto et al.). Es importante tener en cuenta esta característica ya que estas ramas pueden ser interpretadas erróneamente como arterias adicionales en los estudios diagnósticos por imágenes.

Existe poca información sobre el calibre de las arterias renales. Los estudios imagenológicos se centran en casos patológicos, especialmente los asociados a la hipertensión por estenosis renovascular (Holden et al.; Kawamoto et al.). El calibre reportado en estudios previos en un rango de 4,9-5,9 mm (Aytac et al., 2003; Saldarriaga et al.) es similar al observado en el presente caso. Con relación al diámetro de las arterias renales principales y adicionales, Aytac et al. señalan que es muy probable (98,8\% de especificidad) la presencia de arteria renal adicional cuando la arteria renal principal tiene un diámetro menor a $4,1 \mathrm{~mm}$, mientras que en presencia de una arteria renal principal con calibre mayor a 5,5 $\mathrm{mm}$ muy probablemente no habrá ARA.

La expresión morfológica para la irrigación del hí- 
gado descrita en los textos clásicos ha sido reportada en el 55-80 \% de los grupos poblacionales estudiados (Koops et al.; Hiatt et al.). Se caracteriza por la presencia de una arteria hepática común procedente del tronco celiaco, con una longitud de $4 \mathrm{~cm}$ que da origen a las arterias gastroduodenal y hepática propia, quien a su vez asciende por el margen libre del omento menor, delante de la vena porta, a la izquierda del conducto colédoco y cerca del hilio hepático se divide en sus dos ramas terminales derecha e izquierda. Con relación a su origen o destino, a las variaciones de la arteria hepática y sus colaterales se les han denominado como arterias hepáticas aberrantes, las cuales se han dividido en accesorias y reemplazantes. La rama accesoria se distribuye junto a la arteria común para irrigar un mismo lóbulo, y la reemplazante irriga un lóbulo en ausencia de la usual (Hiatt et al.; Ottone et al., 2006).

La presencia de la rama derecha de la arteria hepática originada desde la arteria mesentérica superior, como la observada en este caso, ha sido reportada en el 9,5\% con un rango de 6,2-18 \%. Pero corresponde a la rama hepática izquierda originada desde la arteria gástrica izquierda a la expresión variante de la arteria hepática reportada con mayor incidencia (9,1-30 \%). (Hiatt et al.; Ottone et al.; Koops et al.; Michels; Abdullah et al., 2006). Por la significativa variabilidad en la anatomía de las arterias que irrigan al hígado se hace necesario tener un buen conocimiento de éstas, con el fin de disminuir el riesgo de iatrogenias durante los procedimientos que comprometan al piso supra-mesocólico. En la cirugía de trasplante de hígado se ha reportado una mayor incidencia de complicaciones arteriales en aquellos pacientes a los que se les tuvo que hacer mayor número de anastomosis por la presencia de variaciones anatómicas. Por tal motivo, si bien las variaciones no causan contraindicaciones absolutas, se convierten en estos casos en factor determinante que puede disminuir la posibilidad de un éxito quirúrgico (Ottone et al.)

Las alteraciones de riñón y vías urinarias pueden estar presentes como anomalías congénitas identificadas a edad temprana en el 0,3-0,96\% de los individuos o como variaciones unilaterales y bilaterales asintomáticas identificadas de manera accidental durante la autopsia. Las características más frecuentes de las variaciones que se presentan son ausencia, formación rudimentaria, génesis doble o múltiple del riñón o del sistema colector (Caiulo et al., 2012). La variación del sistema colector descrita para el presente caso corresponde a un uréter bífido unilateral derecho con distribución medial y lateral al hilio renal, que presenta una incidencia de $0,5-3,0 \%$ en casos previamente publicados. Este tipo de variación es tres veces más común que la duplicación completa y su asociación es más frecuente en el riñón derecho que en el izquierdo (Anderson \& Cadeddu, 2012).
Las características morfométricas del riñón derecho que presentó la variación permitieron establecer diferencias con el riñón izquierdo, de tal manera que se observó un aumento en el largo de 22,7 mm y una reducción en el espesor de 2,1 mm, diferencias observadas previamente en los hallazgos identificados por Dinanath et al. (2011). Las características del sistema colector descritas hasta el momento reportan un $10 \%$ de casos con pelvis renal bífida y el $4 \%$ de casos con duplicación ureteral incompleta asociada a pelvis renal doble; nuestro reporte establece un caso de doble uréter unilateral incompleto originado de pelvis renal superior e inferior, con convergencia de los uréteres a los 29,7 $\mathrm{mm}$ de la pared vesical, similar a lo observado por Ghobashy et al., en 2012, pero distante con los $5 \mathrm{~cm}$ identificados por Vaidya et al. (2014). Los calibres de los uréteres bífidos derechos fueron de $3,73 \mathrm{~mm}$ y $3,51 \mathrm{~mm}$, mientras que el calibre del uréter luego de la convergencia fue $4,07 \mathrm{~mm}$, similar a los $4,55 \mathrm{~mm}$ del segmento pélvico del uréter izquierdo, mediciones que permiten establecer que no existen diferencias morfométricas apreciables entre el sistema colector derecho e izquierdo a pesar de la variación.

Los análisis morfométricos de las variantes del sistema colector renal no son frecuentes y su determinación se establece como descripciones cualitativas de la presentación morfológica, por lo tanto los hallazgos descritos en este manuscrito no solo enriquecen los conocimientos del sistema colector sino que a pesar de considerarse al uréter bífido como una variación asintomática permitirá ayudar a esclarecer la causa de posibles complicaciones como la litiasis urinaria, estenosis ureteral o pielonefritis, condiciones patológicas frecuentes con la presencia de anomalías congénitas (Shapiro et al., 2012).

BALLESTEROS, L. E.; ESTUPIÑAN, H. Y. \& BALLESTEROS, D. R. Vascular variations in urinary tract and abdominal cavity. Case report. Int. J. Morphol., 34(1):404-409, 2016.

SUMMARY: Here we present a rare case of multiple abdominal cavity variations in a 50-year-old male cadaveric specimen of the anatomy laboratory of the Universidad Industrial de Santander (Bucaramanga, Colombia). The anatomical dissection revealed arterial variations (right additional renal artery and origin of the right hepatic branch from the superior mesenteric artery), venous (right additional renal vein) and urinary tract (duplicated ureter on the right side). These multiple anatomic variations in addition to raising academic interest, should be considered and described correctly by clinicians while performing surgical, radiological and imaging procedures in the abdominal cavity.

KEY WORDS: Right renal artery; Right renal vein, Right branch of hepatic artery; Ureter bifida. 


\section{REFERENCIAS BIBLIOGRÁFICAS}

Abdullah, S. S.; Mabrut, J. Y.; Garbit, V.; De La Roche, E.; Olagne, E.; Rode, A.; Morin, A.; Berthezene, Y.; Baulieux, J. \& Ducerf, C. Anatomical variations of the hepatic artery: study of 932 cases in liver transplantation. Surg. Radiol. Anat., 28(5):468-73, 2006.

Anderson, J. K. \& Cadeddu, J. A. Surgical Anatomy of the Retroperitoneum, Adrenals, Kidneys, and Ureters. In: Wein, A. J. (Ed.). Campbell-Walsh Urology. 10 ${ }^{\text {th }}$ ed. Philadelphia, Saunders, 2012. pp.3-70.

Aytac, S. K.; Yigit, H.; Sancak, T. \& Ozcan, H. Correlation between the diameter of the main renal artery and the presence of an accessory renal artery: sonographic and angiographic evaluation. $J$. Ultrasound Med., 22(5):433-9, 2003.

Ballesteros, L. E.; Saldarriaga, V. \& Ramirez, L. M. Morphologic evaluation of the renal veins: a study with autopsy material from Colombian subjects. Rom. J. Morphol. Embryol., 55(1):77-81, 2014.

Beckmann, C. F. \& Abrams, H. L. Renal venography: anatomy, technique, applications, analysis of 132 venograms, and a review of the literature. Cardiovasc. Intervent. Radiol., 3(1):45-70, 1980.

Caiulo, V. A.; Caiulo, S.; Gargasole, C.; Chiriacò, G.; Latini, G.; Cataldi, L. \& Mele, G. Ultrasound mass screening for congenital anomalies of the kidney and urinary tract. Pediatr. Nephrol., 27(6):949-53, 2012.

Ciçekcibas,i, A. E.; Ziylan, T.; Salbacak, A.; Seker, M.; Büyükmumcu, M. \& Tuncer, I. An investigation of the origin, location and variations of the renal arteries in human fetuses and their clinical relevance. Ann. Anat., 187(4):421-7, 2005.

Costa, H. C.; Moreira, R. J.; Fukunaga, P.; Fernandes, R. C.; Boni, R. C. $\&$ Matos, A. C. Anatomic variations in vascular and collecting systems of kidneys from deceased donors. Transplant. Proc., 43(1):61-3, 2011.

Dinanath, P.; Ashwini, A.; Annarad, G. \& Nagaraj, S. Bilateral complete duplex renal pelves and ureters - a case report. Int. J. Anat. Var., 4:192-4, 2011.

Ghobashy, A.; El-Shazly, M.; Lari, A.; Al-Hunaidy, O.; Allam, A.; Alenezy, N.; Yordanov, E. \& Hathout, B. A case of complete renal duplex with h-shaped ureter. Case Rep. Urol., 2012:643207, 2012.

Hiatt, J. R.; Gabbay, J. \& Busuttil, R. W. Surgical anatomy of the hepatic arteries in 1000 cases. Ann. Surg., 220(1):50-2, 1994.

Holden, A.; Smith, A.; Dukes, P.; Pilmore, H. \& Yasutomi, M. Assessment of 100 live potential renal donors for laparoscopic nephrectomy with multi-detector row helical CT. Radiology, 237(3):973-80, 2005 .

Janschek, E. C.; Rothe, A. U.; Hölzenbein, T. J.; Langer, F.; Brugger, P. C.; Pokorny, H.; Domenig, C. M.; Rasoul-Rockenschaub, S. \& Mühlbacher, F. Anatomic basis of right renal vein extension for cadaveric kidney transplantation. Urology, 63(4):660-4, 2004.
Kawamoto, S.; Lawler, L. P. \& Fishman, E. K. Evaluation of the renal venous system on late arterial and venous phase images with MDCT angiography in potential living laparoscopic renal donors. A. J. R. Am. J. Roentgenol., 184(2):539-45, 2005.

Khamanarong, K.; Prachaney, P.; Utraravichien, A.; Tong-Un, T. \& Sripaoraya, K. Anatomy of renal arterial supply. Clin. Anat., 17(4):334-6, 2004.

Koops, A.; Wojciechowski, B.; Broering, D.; Adam, G. \& KrupskiBerdien, G. Anatomic variations of the hepatic arteries in 604 selective celiac and superior mesenteric angiographies. Surg. Radiol. Anat., 26(3):239-44, 2004.

Michels, N. A. Newer anatomy of the liver and its variant blood supply and collateral circulation. Am. J. Surg., 112(3):337-47, 1966.

Ottone, N. E.; Arrotea Molina, A.; Domínguez, M. L.; Lo Tartaro, M.; García de Quiros, N.; Medan, C.; Blasi, E. \& Bertone, V. H. Aberrants hepatic arteries. Research over 64 disections. Int. J. Morphol., 24(4):581-5, 2006.

Saldarriaga, B.; Pinto, S. A. \& Ballesteros, L. E. Morphological expression of the renal artery. A direct anatomical study in a Colombian half-caste population. Int. J. Morphol., 26(1):31-8, 2008 .

Satyapal, K. S.; Haffejee, A. A.; Singh, B.; Ramsaroop, L.; Robbs, J. V. \& Kalideen, J. M. Additional renal arteries: incidence and morphometry. Surg. Radiol. Anat., 23(1):33-8, 2001.

Satyapal, K. S. The renal veins: a review. Eur. J. Anat., 7 Suppl. 1:43-52, 2003.

Shapiro, E.; Bauer, S. B. \& Chow, J. S. Anomalies of the Upper Urinary Tract. In: Wein, A. J. (Ed.). Campbell-Walsh Urology. 10th ed. Philadelphia, Saunders, 2012. pp.3123-60.

Vilhova, I.; Kryvko, Y. Y. \& Maciejewski, R. The radioanatomical research of plural renal arteries. Folia Morphol. (Warsz), 60(4):337-41, 2001.

Vaidya, Y. P.; Waghmare, J. E.; Tarnekar, A. M. \& Shend, M. R. Unilateral isolated incompletely duplicated ureter. J. Mahatma Gandhi Inst. Med. Sci., 19(2):148-50, 2014.

Zhu, J.; Zhang, L.; Yang, Z.; Zhou, H. \& Tang, G. Classification of the renal vein variations: a study with multidetector computed tomography. Surg. Radiol. Anat., 37(6):667-75, 2015.

\section{Correspondencia: \\ Dr. Luis Ernesto Ballesteros \\ A. Avenida González Valencia \#54.25 Apto 404. \\ Bucaramanga \\ COLOMBIA}

Email: Iballest56@yahoo.es

Recibido : 14-10-2015

Aceptado: $22-12-2015$ 